\title{
Estrategia educativa para la participación de los padres en compromisos escolares
}

\section{Education strategy for the involvement of parents in school commitments}

\author{
Virna Rosa julio Tuesca \\ Magister Universidad Autónoma del Caribe. Psicóloga egresada de la universidad Simón Bolívar. Docente de la IED Rural de Palmira- Pueblo viejo \\ Magdalena,Virnaros@hotmail.com.
}

Milena Maritza Mánuel Girón

Magister Universidad Autónoma del caribe. Psicopedagoga egresada de la C.U.C. Docente I.E.D. San José de Pueblo Viejo Magdalena, Milemmg@hotmail.com

Luis Ricardo Navarro Díaz

Doctorando en Ciencias Sociales Universidad del Norte, Magister en Comunicación Universidad del Norte, docente Universidad Tecnológica de Bolívar y docente Universidad Autónoma del Caribe, Rnavarrod@hotmail.com

Recibido: Oct 30 de 2012

Aceptado: Nov 22 de 2012

\section{RESUMEN}

El artículo presenta los resultados más relevantes del trabajo de investigación de maestría en educación, la cual tuvo como objetivo diseñar una estrategia educativa para la participación de padres de familia en los compromisos escolares de los estudiantes de la I.E.D. Rural de Palmira, Pueblo-Viejo, Magdalena". El universo de estudio estuvo conformado por 522 estudiantes, 420 padres de familia y 10 docentes, para un total de 1190 personas, de la cual se tomó como muestra al grado quinto, conformado por 22 padres de familia, 22 estudiantes con edades entre 10 y 13 años y 10 docentes de la Institución para una muestra total de 54 personas a las cuales se les aplicaron los instrumentos de recolección de información. Se construyó un cuadro matriz de análisis, que cruza cada una de las categorías con lo que dice cada actor social sobre ellas.. La participación de docentes, padres y estudiantes, con enfoque constructivista, fue vital para el diseño de la propuesta, centrada en un plan de acción, como estrategia educativa, a través de cuatro ejes temáticos a saber: cualificación a padres de familia, fortalecimiento de niveles de participación, fortalecimiento de canales de comunicación, normatividad sobre participación. La propuesta fue originada en el diagnóstico y la necesidad de búsqueda participativa de contribuir al mejoramiento de la participación de los padres en los compromisos escolares.

Palabras Clave: Educación, Estrategia Educativa, Participación, Compromisos Escolares.

\begin{abstract}
This article presents the most relevant results of the research of master's degree in education, which aimed to design an education strategy for the participation of parents in the school of students from the Rural European Institute of Palmira, Pueblo-Viejo, Magdalena commitments". The universe of study was formed by 522 students, 420 parents and 10 teachers, for a total of 1190 people, which was taken as a sign to the fifth grade, consisting of 22 parent, 22 students aged between 10 and 13 years and 10 teachers of the institution for a total sample of 54 people which we applied the instruments for collecting information. Built a matrix table of analysis, which crosses each of the categories with what each social actor on them.. The participation of teachers, parents and students, with constructivist approach, was vital for the design of the proposal, focusing on a plan of action, as educational strategy, through four themes namely: qualification to parents, strengthening levels of participation, strengthening of channels of communication, regulations on participation. The proposal originated in the diagnosis and the participatory search, whit need to contribute to the improvement of the participation of parents in school commitments
\end{abstract}

Key Words: Education, Strategy Education, Participation, school commitments 


\section{Introducción}

El derecho y el deber de la educación de los niños son para los padres de familia primordial e inalienable. Por ello es hora que vuelvan su mirada a la escuela y participen en los compromisos escolares que ésta ofrece al educando, para compartir su experiencia y juntos dialogar en busca de cambios tanto para la familia, como para el menor estudiante. La escuela activa, se ha iniciado a partir de las innovaciones que están presentes e involucran la participación de todos los miembros de la comunidad educativa, mediante el diálogo, la escucha, la reflexión en equipo, el debate, los consensos y la toma de decisiones en trabajo de carácter cooperativo, situación afirmada por Mogollón (2011) p.5.

Martínez afirma que aunque se ha debatido mucho sobre la necesidad de integración de la comunidad educativa, lo cierto es que la educación se ha planeado, programado, administrado e impartido, al margen de los padres de familia, quienes en su gran mayoría, ignoran el tipo de participación que la escuela espera de ellos.

González (2012) sostiene que esta situación ha impactado en la educación, con cambios en la sociedad y en las instituciones educativas, en donde la crisis de las familias, la situación actual del país y la difícil tarea en la labor docente, hace necesario conformar una labor sólida que contribuya a resignificar la verdadera función de la escuela, de la vida familiar y social, para que ésta conlleve a la buena formación del educando.

Sobre la importancia de la participación de los padres de familia en el sistema educativo se hace énfasis en la publicación número 4 de Contactos del Centro de documentaciones de (Unicef - Cide. 2008), aquí se afirma que la educación sólo puede realizarse plenamente cuando la familia y la escuela trabajan juntas. La familia, como medio natural de desarrollo del hombre, y la escuela, como institución social encargada específicamente de educar, deben cooperar en forma conjunta, a favor siempre, de niños y jóvenes.

Les han atribuido en exclusividad a las familias el rol de satisfacer las necesidades biológicas, afectivas y de formación valórica. A la escuela, en cambio, se le considero una institución destinada a la satisfacción de necesidades intelectuales y académicas, Gallardo, Gonzalo. (2011), p. 2, en su ensayó Familia-escuela. Esta misma apreciación es presentada por Arancibia, Herrera, Strasser (1999) p.190 Pero actualmente las experiencias vividas en la educación, han demostrado que el trabajo en conjunto a favor de la educación de un niño es mucho más eficiente, y que cuando se educa en alianza colegio/familia, acompañándolo en toda su realidad, se logra un desarrollo más armónico e integral del individuo.

La familia es el primer contexto de aprendizaje para las personas, en este sentido, es importante aclarar que en su seno aprenden no sólo los niños sino también los adultos, asílo afirmó González Tornaría en el foro sobre Educación y Valores Democráticos (2000).
La necesidad de promocionar la participación de los padres, ha ido creciendo de manera sistemática con el pasar de los años Morales, (1998); Gubbins,( 2001); Organización de las Naciones Unidas para la Educación, la ciencia y la cultura [UNESCO], (2004). Por lo tanto, para lograr que los padres de familias, lleguen a participar, es preciso poner en práctica un plan de trabajo sistemático que gradualmente los involucre en los compromisos escolares que los hijos adquieren en su formación.

En la Institución Educativa Rural, ubicada en el corregimiento de Palmira, municipio de Pueblo Viejo- Magdalena, lugar donde se realizó esta investigación, hay que reconocer el intento por vincular a los padres de familia mediante mecanismos tales como: charlas, reuniones o llamados al plantel y no ha sido posible lograr una vinculación activa en los compromisos escolares que sus hijos deben cumplir en la escuela, por lo tanto la interacción docente - estudiante - acudiente está disociada, puesto que los padres se muestran indiferentes ante el deber que adquieren con el plantel especialmente en la función de orientación y colaboración en los compromisos escolares de sus acudidos.

La presencia de los padres de familias en la institución en especial aquellos que hacen parte del grado quinto es mínima, y su accionar solo se limito al diligenciamiento del proceso de matrícula, y algunas veces en la recepción de informes académicos, cuando se les solicita en forma reiterada pero en ocasiones no asisten porque sus actividades laborales centradas en la pesca o la economía del rebusque la consideran de mayor importancia, teniendo en cuenta que es el principal medio de subsistencia para mantener a su familia. (Información dada por la directora del plantel).

La actitud evidenciada en los padres de familia que hacen parte del grado quinto en la escuela de Palmira, se aprecia en la falta de orientación que brindan a los niños al cumplir los compromisos escolares y la escasa presencia de ellos en la escuela, quienes en pocas ocasiones se acercan a preguntar sobre la forma como se está desenvolviendo el hijo en el proceso educativo y aunque se les ha hablado, explicado y orientado sobre la forma de guiar al menor pocos padres aplican estas recomendaciones afectando de una u otra forma el trabajo de los docente y la calidad de la educación que se le quiere brindar al estudiante en la institución.

La anterior descripción, llevó a plantear el siguiente interrogante: ¿Qué estrategia educativa se podrán diseñar para la participación de los padres de familia en los compromisos escolares de los estudiantes de quinto grado pertenecientes a la I.E.D. Rural de Palmira, Pueblo Viejo - Magdalena?

Con esta investigación se hace un aporte a la educación en el corregimiento de Palmira, sensibilizando al padre de familia para que cumpla su misión de ayudar en los compromisos educativos de sus hijos, y al docente para que en forma conjunta, dirija, enseñe y desarrolle los procesos de formación, haciendo del niño el hombre que se desea para el futuro, pues la constante relación hogar, escuela, padre de familia, profesores, son necesarias para 
ayudar en la tarea de formación de hombres nuevos que necesita dicha comunidad del corregimiento de Palmira, y así "cumplir con el deber de asistir y proteger a los niños para garantizarles sus derechos y el desarrollo armónico e integral", Congreso de la república. Ley General de Educación (Ley 115 de 1994).

A partir de la promulgación de la Constitución Nacional de 1991, se parte la historia de la Educación en Colombia, generando en nuestro país una transformación en los diferentes estamentos educativos y muy especialmente en lo relacionado con la familia la cual ha sido profundamente beneficiada ya que la nueva legislación pone a su alcance, varias acciones con el fin de proteger sus derechos, velar por el cumplimiento de sus deberes, en búsqueda del desarrollo integral del ser humano. Sin embargo no se puede desconocer los esfuerzos que a través de la historia se han llevado a cabo para el mejoramiento de la Educación, contando con la participación de los padres de familias.

Fue así como a través del decreto 088 de 1976, el Gobierno Nacional planteó la necesidad de un cambio en el sistema de evaluación escolar, abriendo con ello el camino para la promoción del alumno con la ayuda conjunta de padres de familia. Dando continuidad a las reformas, se dio, desde 1975 a 1978, el plan " Para Cerrar la Brecha" del departamento nacional de planeación, donde se identifica como uno de los problemas del sistema educativo colombiano el de la despreocupación de los padres por la educación de los hijos y una de las políticas para su solución fue la información y motivación, de los padres de familia como una forma de retención de los niños en la escuela.

Siguiendo el orden de ideas en lo que tiene que ver con la participación, fue evidente, el "Plan para la Erradicación de la Pobreza Absoluta (1986 a 1990)", donde se integra a los padres de familia y a la comunidad educativa para estimular la labor docente, colaborar con sus experiencias en la labor educativa, para que se amplíe la relación escuela - hogar y escuela - comunidad; creándose a través del decreto 2272 de( 1998), la jurisdicción de familia y la Ley 134/94 reglamenta lo relacionado con los mecanismos de participación ciudadana.

Las disposiciones constitucionales al trasladar a la rama legislativa, la facultad de regular la Educación, permitieron una mayor participación de la sociedad, haciendo de la Educación no solo un derecho fundamental, sino un servicio publico con función social.

Esta participación escolar es entendida como el poder real de tomar parte activa en la elaboración y desarrollo del proceso educativo, tanto a nivel micro como macro social, de todos los que intervienen en él: docentes, alumnos y apoderados Gallardo y Calisto, (2004), presentando objetivos finales claros y conscientes, o metas no conscientes, pero significativos para el sistema social Navarro, (2002). Según este autor la participación no es solamente una representación ni una ideología, sino un fenómeno objetivable y operacionalizable mediante indicadores, compuesto de actividades voluntarias a través de las cuales los miembros de una comunidad escolar intervienen directa o indirectamente en las actividades relacionadas con el proceso educativo.

Existen diferentes posturas respecto a los alcances de la participación, quienes promueven un rol más activo de las familias en términos de los procesos de aprendizaje de los niños en el hogar o en las escuelas; y quienes promueven la participación organizada de los padres en la gestión educativa y en el control de la eficacia del sistema Gubbins, (2001); Navarro, (2002).

Sumado a lo anterior, también se debe tener presente que la participación no es un concepto unitario, ya que contiene dos dimensiones: el Área o contenido del aprendizaje en que toman parte o participan (académico v/s no académico), y la Institución donde se toma parte o participa (hogar v/s escuela, a partir de las que es posible establecer cuatro áreas donde puede darse la participación: En el hogar y en el ámbito académico, en el hogar y en ámbito no académico, en la escuela y en el ámbito académico, y en la escuela y en el ámbito no académico Alarcón, (2002).

Por otro lado, las dificultades para la participación de los padres incluirían la falta de preparación académica de los padres o su preocupación por problemas familiares más apremiantes (según los directores).

La participación de los padres en la vida escolar parece tener repercusiones tales como: Una mayor autoestima de los niños y niñas. Un mejor rendimiento escolar. Mejores relaciones padres/ madres e hijos/hijas. . Y Actitudes de los padres y madres hacia la escuela. Los efectos repercuten incluso en el profesorado, ya que los padres y madres consideran que los más competentes son aquellos que trabajan con la familia Pineault (2001)

Si partimos del hecho de que la familia en la educación ha ido evolucionando con la incorporación de la mujer al mundo laboral y con otros cambios de diversa índole, sobre todo político y económico. La familia ha pasado de ser la encargada de la formación de sus hijos, a delegar en la escuela parte de esta tarea. Esta situación demanda una nueva visión educadora de la familia y la escuela, los cuales deben trabajar unidos en un proyecto común.

Una correcta educación comienza desde el nacimiento. Por ello, desde que el niño y la niña entran a la escuela, se solicita a sus padres colaboración en determinadas actividades Esta colaboración cuando se termina la educación desaparece casi en la mayoría de los casos. Si la participación de los padres se consolida bien en la primera etapa de la educación, no tiene que desaparecer una vez los niños y niñas pasan a primaria. Padres y madres tienen responsabilidades compartidas en la ecuación de los hijos/as, por lo que es imprescindible que haya un contacto entre ambos. Palacios y oliva (1991)

En cuanto al papel de la familia y la escuela en la educación actual, Martínez Torres, (2011), de la revista actualidad educativa, enfatiza, en que es necesaria una forma de enfocar la educación en 
la familia, y la necesidad de su participación en ámbitos sociales. La sociedad ha sufrido una evolución en los últimos tiempos que ha repercutido en la familia y la escuela. Tanto es así que uno de los temas más destacados respecto a la educación en estos días es la colaboración entre ambas.

La participación de los padres en la educación de sus hijos es importante. Cuando los padres participan en su enseñanza, por lo general los hijos obtienen mejores resultados en su proceso educativo, tienen un mejor comportamiento, y alcanzan actitudes más positivas hacia la escuela y crecen para ser más exitosos en la vida.

\section{Metodología}

Por las características de esta investigación, se trabajó con el paradigma "Socio-crítico", para hacer posible el análisis de una realidad social que puede ser intervenida a través de ofrecimiento de una respuesta como aporte a la solución y para este caso consistió en el diseño de una Estrategia educativa, para la participación activa de los padres de familias.

Este paradigma en el ámbito socioeducativo, reconoce la presencia de imperativos sociales que van construyendo la realidad. Autores como Stenhouse, Giroux, Kemmis, Apple, Carr, entre otros; son dignos representantes de este paradigma que tiene sus orígenes en la teoría comunicacional de Jürgen Habermas y la escuela crítica alemana de Frankfurt Giroux, (1992). Edgar Morín, sin duda es considerado como su gran teórico, lanza un desafío cuando dice: "Tratemos de ir no de lo simple a lo complejo, sino de la complejidad hacia aún más complejidad. Lo simple, repitámoslo, no es más que un momento, un aspecto entre muchas complejidades" Morín (2000)

Es así, como se consideró trabajar con el paradigma "Socio-crítico", que a partir del análisis de una realidad social pudiera ser intervenida a través de ofrecimiento de una respuesta con un conjunto de actividades, en el entorno educativo, incorporadas en el diseño de un plan de acción, como estrategia educativa para padres para lograr de forma eficaz y eficiente la consecución de los objetivos educativos esperados. Este diseño permite la construcción colaborativa, con acciones realizado tanto por el docente como por el estudiante, los cuáles pueden retroalimentarse mutuamente Matus, O. (2009), p.11

Este tipo de estudio, permitió clarificar el problema, hacer el diagnóstico y diseñar la propuesta de cambio. Además, se desarrolló bajo el enfoque cualitativo, puesto que favorece más los estudios en educación y conlleva procesos inductivos, generativos, constructivos y subjetivos. La metodología cualitativa se interesa por capturar y descubrir significados una vez que el investigador se sumerge en los datos. Buendía Eximan, Colás Bravo y Hernández Pina(1999) P.261

Esta investigación se desarrolló en la IED Rural de Palmira - Pueblo Viejo, Magdalena, con un universo de estudio conformado por
522 estudiantes, 420 padres de familia y 10 docentes, para un total de 1190 personas, de la cual se tomó como muestra al grado quinto, conformado por 22 padres de familia, 22 estudiantes con edades entre 10 y 13 años y 10 docentes de la Institución para una muestra total de 54 personas a las cuales se les aplicaron los instrumentos de recolección de información.

Una vez aplicados los instrumentos, se procedió a tomar las categorías de análisis de la investigación estrategias educativas, participación y compromisos escolares, ubicándolas en un cuadro matriz de análisis, que cruza cada una de las categorías con lo que dice cada actor social sobre ellas, (Ver fig. 1) a partir de este cuadro matriz elaborado, se procedió a realizar el análisis e interpretación de los resultados de las entrevista y observación directa( ver fig. 2), aplicada a los integrantes de la comunidad educativa, conformada por los padres de familias, docentes y estudiantes pertenecientes al grado quinto, teniendo en cuenta cada una de las categorías de análisis, con sus correspondientes preguntas, además de tener en cuenta los diferentes autores utilizados en el marco teórico y también la apreciación del grupo investigador.

La necesidad de promocionar la participación de los padres en la institución, ha ido creciendo de manera sistemática con el pasar de los años Morales, (1998); Gubbins, (2001), son enfáticos en afirmar que para lograr que los padres de familias, lleguen a participar, es preciso poner en práctica un plan de trabajo sistemático que paulatinamente vaya involucrándolo en los compromisos escolares que los hijos adquieren en su formación.

Son variadas las formas como los padres de familia ayudan a sus hijos en los compromisos escolares, todas serian ideales siempre y cuando se tradujeran en resultados positivos, en lo que tiene que ver con la participación del padre de familia en los compromisos escolares de sus hijos. Entre las estrategias más frecuentes y utilizadas por los padres de familia, se destacan las siguientes:

- Aclaran las dudas que el estudiante pueda tener, y si ellos no lo comprenden, buscan la explicación de otro padre de familia.

- Compran materiales para la elaboración de algunas tareas, si es necesario.

- Buscan libros o enciclopedias.

- Le dan el dinero para que investiguen en internet.

- Revisan los compromisos y vigilan que el estudiante los realice.

Resulta preocupante que en ocasiones el nivel de responsabilidad de los padres de familia con la educación de sus hijos sea bajo, puesto que solo se limitan a matricularlos en la escuela, en ocasiones adquieren los útiles escolares, pero no realizan un acompañamiento a todo el proceso educativo del niño. En algunos casos dejan la responsabilidad de asistir al colegio en los mismos estudiantes, es decir, si ellos no quieren asistir a clases no lo hacen y los padres lo permiten sin ningún problema. 
El diseño de la matriz propuesta, como fundamento estratégico, para subsanar las falencias resultantes del diagnóstico, se puede apreciar en la fig. 3 .

Por su parte, aspectos de la problemática se clarificó con la siguiente matriz de observación:

Es así, como se encontró, que los padres consideran que si tienen participación en las actividades que se realizan en la institución educativa, y que está acorde con su responsabilidad, pero una muestra de observación lo contradice, ya que al ser convocados a reuniones de cualquier carácter en forma general, su nivel de participación es escaso. Esta situación fue corroborada con base a las reuniones que se han desarrollado durante el año lectivo.

\section{Resultados y discusión}

Existe una escasa participación de las familias pues solo participan algunos del total de padres que componen las comunidades educativas. Sin embargo, esta percepción no la comparten los padres, quienes consideran que participan en las diferentes actividades de la institución educativa. La explicación a esta diferencia podría estar en que la respuesta que da el profesorado corresponde a la percepción que tienen sobre la participación de los padres en general, como colectivo, mientras que la opinión dada por cada padre y madre es sobre su propia actuación.

La frecuencia con que participan las familias depende principalmente de la aparición o no de problemas relacionados con sus hijos. Cuando los padres participan como colectivo, lo hacen principalmente en reuniones en el aula con el profesor, o convocadas por las directivas.

De los tres contextos de participación, el político, académico y comunitario, es este último el que se le ha atribuido históricamente a los padres, seguido del político, a través de su representación en el Consejo Escolar. Por último, el contexto académico relacionado con el proceso de enseñanza-aprendizaje es donde la intervención de los padres está menos definida, no teniéndose claro el cómo y en dónde pudieran intervenir.

Para el profesorado, la mayoría de las familias, simplemente quieren permanecer informadas de todo lo relacionado con la educación de sus hijos olvidándose que además de ello, hay que participaren debates y aportar sugerencias sobre la marcha de la institución educativa.

Fig. 1 Matriz de análisis de la observación.

\begin{tabular}{|c|c|}
\hline ASPECTOS A OBSERVAR & SITUACIONES OBSERVADAS \\
\hline $\begin{array}{l}\text { 1. Estrategias empleadas por } \\
\text { el docente para que el padre de } \\
\text { familia haga presencia en la Ins- } \\
\text { titución }\end{array}$ & $\begin{array}{l}\text { - Llamados verbales, para alguna queja académica y/o comporta mental del hijo. } \\
\text { - } \text { Cotas en los cuadernos de los estudiantes. } \\
\text { - Reuniones académicas por periodos. solo asisten las madres. Y frecuentan las mismas siempre } \\
\text { - Llamados para renovar documentos en las carpetas de los estudiantes. } \\
\text { - Llamados a participar en actividades organizadas por la IED como: pulguero, día de los ancianos. } \\
\text { - Reunión para iniciar el año escolar. } \\
\text { - Reunión para acordar aspectos como: otorgar permiso a sus hijos para participar de algún paseo progra- } \\
\text { mado por la IED. Detalles para la ceremonia de grados. }\end{array}$ \\
\hline $\begin{array}{l}\text { 2. Frecuencia con que el padre } \\
\text { de familia asiste a la Institución }\end{array}$ & $\begin{array}{l}\text { - Solo asisten a la institución cuando son llamados por el docente o convocados por la institución. } \\
\text { - Si alguien peleó o maltrató a su hijo, pero con la intención de defenderlo. }\end{array}$ \\
\hline $\begin{array}{l}\text { 3.Las formas como los padres de } \\
\text { familia ayudan a sus hijos en los } \\
\text { compromisos escolares }\end{array}$ & $\begin{array}{l}\text { - Aclarando las dudas que el estudiante pueda tener, y si ellos no lo comprenden, buscan la explicación de } \\
\text { otro padre de familia. } \\
\text { - Comprando materiales para la elaboración de algunas tareas, si es necesario. } \\
\text { - Buscando libros o enciclopedias. } \\
\text { - Le dan el dinero para que investiguen en internet. } \\
\text { - Revisan los compromisos y vigilan que el estudiante los realice. }\end{array}$ \\
\hline $\begin{array}{l}\text { 4. Nivel de responsabilidad de los } \\
\text { padres de familia con la educación } \\
\text { de sus hijos }\end{array}$ & $\begin{array}{l}\text { - Nivel de responsabilidad es mínimo. } \\
\text { - Los padres se limitan a matricular a sus hijos en la escuela, } \\
\text { - No realizan un acompañamiento a todo el proceso educativo de sus hijos. } \\
\text { - Hay casos que dejan la responsabilidad de asistir al colegio en los mismos estudiantes. }\end{array}$ \\
\hline $\begin{array}{l}\text { 5. La participación de los padres } \\
\text { en las actividades que realizan en } \\
\text { la Institución Educativa }\end{array}$ & - Muy baja la participación a pesar de invitarles por diferentes medios \\
\hline
\end{tabular}

Fuente: Los autores 
El concepto que tienen las familias sobre lo que significa su participación, esta fundamentado en que participar implica permanecer informadas sobre la marcha del aprendizaje de sus hijos y sobre otros aspectos relacionados con el funcionamiento de la institución educativa. Sin tener en cuenta aspectos como el apoyo en las tareas escolares de sus hijos, asistencia puntual y frecuente a la institución, colaborar y asistir a las actividades programadas, y formar parte del gobierno escolar.

Se ha demostrado que cuando se trata de hablar de lo que significa la participación educativa, se dan diferencias según cual sea el sector que participe. El profesorado piensa que la participación educativa conlleva más intervención en la toma de decisiones cuando se trata de su sector que cuando se refiere a la participación de las familias y, por su parte, las familias asumen que la intervención en la toma de decisiones que tiene que ver con todo lo relacionado con la gestión de la institución es competencia del profesorado más que de su sector.

La participación del padre de familia en los compromisos educativos de sus hijos, se hace más eficiente en la medida que este más cercano a la institución educativa.

Esta participación implica aspectos como por ejemplo: ir a dejar o a buscar a los estudiantes a la, institución lo que permite tener un contacto con el entorno educativo, así como también, la posibilidad de acercarse a conversar con el profesor para saber y estar al tanto de aspectos como el comportamiento, el rendimiento académico y de las diferentes actividades que se desarrollan en la institución. También incluye la colaboración en actividades como (jean day, bingos, rifas) y formar parte del gobierno escolar.
La participación de los padres puede ayudar a mejorar la calidad de los sistemas escolares públicos y brindar un mosaico de oportunidades para que sus hijos tengan éxito en su tránsito por la escuela. Machen y Notar (2005).

Es a partir de una relación entre escuela y padres de familia y de la participación, de los últimos que se pueda contribuir a la formación de mejores ciudadanos, (Morín, 2001). Los padres de familia deben cumplir con el deber de asistir y proteger a los niños para garantizarles sus derechos y el desarrollo armónico e integral. (Ley General de Educación)

Seguidamente, se muestra la matriz que sirvió de derrotero, para el análisis y discusión arriba planteados, ver fig. 2

Teniendo en cuenta el objetivo general de la investigación que consistió en el diseño una estrategia educativa para la participación de padres de familia en los compromisos escolares de los estudiantes de quinto grado de la I.E.D. Rural de Palmira, Pueblo-Viejo, Magdalena" y dando cumplimiento a la normatividad existente, se ofrece a los padres de familia la presente propuesta, con el objetivo de contribuir a la participación de estos en el proceso de formación de sus hijos.

Lo anterior surge del análisis realizado a los resultados de los instrumentos aplicados en la Institución lo cual conduce a afirmar que los padres de familia se encuentran ajenos al conocimiento de su papel como formadores, dificultando así la educación de sus hijos, por lo que se hace necesario brindarles una orientación que contribuya a la solución de la problemática.

\section{Fig. 2 Matriz de análisis de la entrevista según categorías y estamentos}

\begin{tabular}{|c|c|c|c|}
\hline Categorías / Preguntas & Docentes & Estudiantes & Padres de familias \\
\hline $\begin{array}{l}\text { CATEGORIA: ESTRATEGIA } \\
\text { EDUCATIVA } \\
\text { 1. ¿Cómo es la comunicación } \\
\text { que mantienen entre docentes } \\
\text { y padres de familias de los es- } \\
\text { tudiantes? }\end{array}$ & $\begin{array}{l}\text {-Muy buena la comunicación } \\
\text {-Cuando se necesita hablar con } \\
\text { ellos acuden puntualmente } \\
\text {-Siempre hay una comunicación } \\
\text { clara y precisa } \\
\text {-Regularmente están presente } \\
\text { en la institución al llamárseles } \\
\text {-Con solo un llamado ellos acu- } \\
\text { den a la institución. }\end{array}$ & $\begin{array}{l}\text {-Muy buena comunicación y cons- } \\
\text { tante } \\
\text {-Escasa porque el trabajo les im- } \\
\text { pide ir a la institución } \\
\text {-Regular porque solo llegan al co- } \\
\text { legio cuando quieren saber algo } \\
\text { sobre el estudiante } \\
\text {-Solo se comunican mediante no- } \\
\text { tas o cuando los mandan a llamar. }\end{array}$ & $\begin{array}{l}\text {-Solo hay comunicación cuando asisto } \\
\text { voluntariamente a la institución } \\
\text {-Cuando les llaman al plantel } \\
\text {-Regular, solo cuando hay necesidad se } \\
\text { comunican con los docentes } \\
\text {-Siempre están presente } \\
\text {-La comunicación se da por intermedio } \\
\text { del niño }\end{array}$ \\
\hline $\begin{array}{l}\text { 2. ¿Cuál es la estrategia uti- } \\
\text { lizada por la institución para } \\
\text { invitar a los padres de familias } \\
\text { a participar activamente de las } \\
\text { actividades académicas que se } \\
\text { realizan? }\end{array}$ & $\begin{array}{l}\text {-Información escrita con los } \\
\text { niños } \\
\text {-avisos publicitarios a través } \\
\text { de carteleras } \\
\text {-Invitación verbal } \\
\text {-A través de reuniones progra- } \\
\text { madas para comprometerlos en } \\
\text { actividades. }\end{array}$ & $\begin{array}{l}\text {-Llamándolos a reuniones en el } \\
\text { colegio } \\
\text {-Mediante informaciones llevadas } \\
\text { por los niños } \\
\text {-A través de comunicación verbal } \\
\text {-Invitaciones a eventos en la ins- } \\
\text { titución-Invitaciones a reuniones }\end{array}$ & $\begin{array}{l}\text {-Sólo hay comunicación cuando asisto } \\
\text { voluntariamente a la institución } \\
\text {-Cuando les llaman al plantel } \\
\text {-Regular, solo cuando hay necesidad se } \\
\text { comunican con los docentes2 } \\
\text {-Siempre están presente } \\
\text {-La comunicación se da por intermedio } \\
\text { del niño }\end{array}$ \\
\hline
\end{tabular}




\begin{tabular}{|c|c|c|c|}
\hline Categorías / Preguntas & Docentes & Estudiantes & Padres de familias \\
\hline $\begin{array}{l}\text { 3. ¿Diga cuáles acciones debe } \\
\text { realizar la institución para que } \\
\text { los padres se vinculen a ella y } \\
\text { colaboren en los compromisos } \\
\text { escolares de sus hijos? }\end{array}$ & $\begin{array}{l}\text {-Manteniendo una relación } \\
\text { constante con los padres de f. } \\
\text {-Invitando al padre de familia a } \\
\text { participar en los proyectos que } \\
\text { se desarrollan } \\
\text {-Convocándolo a las reuniones, } \\
\text { a través de invitaciones perso- } \\
\text { nalizadas. } \\
\text {-Invitándolo a eventos de la } \\
\text { escuela } \\
\text {-Organizando grupos padres. } \\
\text {-Invitarlos a participar, en las } \\
\text { actividades que se planifiquen }\end{array}$ & $\begin{array}{l}\text { Manteniendo una relación cons- } \\
\text { tante con los padres de familias. } \\
\text {-Invitando al padre de familia a } \\
\text { participar en los proyectos que } \\
\text { se desarrollan } \\
\text {-Convocándolo a las reuniones, } \\
\text { a través de invitaciones perso- } \\
\text { nalizadas } \\
\text {-Invitándolo a eventos dela escuela } \\
\text {-Organizando grupos de padres. } \\
\text {-Invitarlos a participar, en lo pla- } \\
\text { nificado }\end{array}$ & $\begin{array}{l}\text {-Manteniendo una relación constante con } \\
\text { los padres de familias. } \\
\text {-Invitando al padre de familia a participar } \\
\text { en los proyectos que se desarrollan } \\
\text {-Convocándolo a las reuniones, a través } \\
\text { de invitaciones personalizadas. } \\
\text {-Invitándolo a los eventos de la escuela } \\
\text {-Organizando grupos de padres. } \\
\text {-Invitarlos a participar, en las actividades } \\
\text { que se planifiquen }\end{array}$ \\
\hline $\begin{array}{l}\text { CATEGORIA: PARTICIPACION } \\
\text { 4. ¿En cuáles actividades acadé- } \\
\text { micas, desarrolladas en la insti- } \\
\text { tución educativa se ha contado } \\
\text { con la participación de padre } \\
\text { de familia? }\end{array}$ & $\begin{array}{l}\text {-Entrega de informe académico. } \\
\text {-Eventos deportivos, sociales } \\
\text { y culturales } \\
\text {-Tienda escolar } \\
\text {-Venta de ropas pulgueros }\end{array}$ & $\begin{array}{l}\text { Entrega de informes académicos } \\
\text {-Reuniones de padres de familias } \\
\text {-Pulgueros } \\
\text {-Festividades de la institución }\end{array}$ & $\begin{array}{l}\text { Solo participan de actividades programadas } \\
\text {-No acostumbran a participar en actividades } \\
\text {-Solo asisten a reuniones académicas } \\
\text {-Solo a entrega de boletines } \\
\text {-En todas las actividades que realiza la } \\
\text { institución educativa }\end{array}$ \\
\hline $\begin{array}{l}\text { 5. ¿Cómo participan los padres } \\
\text { de familias en la realización de } \\
\text { las tareas escolares de sus hijos } \\
\text { dejadas por los maestros? }\end{array}$ & $\begin{array}{l}\text {-Explicaciones breves } 80 \% \text { ile- } \\
\text { trados } \\
\text {-Pocas veces le prestan ayudas } \\
\text {-Las niñas en general no hacen } \\
\text { tareas } \\
\text {-La mayoría de las tareas no se } \\
\text { realizan en casa } \\
\text {-Su nivel académico les dificulta } \\
\text { ayudar en las tareas }\end{array}$ & $\begin{array}{l}\text {-Mi mama me explica y realizo las } \\
\text { tareas que me piden en la escuela } \\
\text {-Me guían en lo que voy a realizar } \\
\text { y lo hago } \\
\text {-Me explican mis padres y procedo } \\
\text { a realizarlas } \\
\text {-Se sientan conmigo para expli- } \\
\text { carme } \\
\text {-Hago las actividades académicas } \\
\text { solo }\end{array}$ & $\begin{array}{l}\text {-Les pregunta y explican lo que deben hacer } \\
\text {-Hacen sus actividades solos } \\
\text {-Le revisan cuadernos y ayudan en las } \\
\text { tareas } 5 \\
\text {-Se sientan con los hijos y hacen las tareas } \\
\text {-Solo en caso de dificultad buscan ayudas } \\
\text {-Preguntan si dejaron actividad y cola- } \\
\text { boran1 } \\
\text {-No ayudan por su nivel académico }\end{array}$ \\
\hline $\begin{array}{l}\text { 6. ¿Regularmente, quién invierte } \\
\text { más tiempo con los hijos cola- } \\
\text { borándole en las actividades } \\
\text { escolares, asistiendo a reunio- } \\
\text { nes en la escuela o visitando al } \\
\text { maestro? }\end{array}$ & $\begin{array}{l}\text {-Las madres de familias } \\
\text {-Tíos } \\
\text {-Padres de familias } \\
\text {-Personas particulares } \\
\text {-Hermanos }\end{array}$ & $\begin{array}{l}\text { Las madres de familias } \\
\text {-Los padres de familias } \\
\text {-Algunos tíos } \\
\text {-Hermanos } \\
\text {-Familiares y amigos allegados }\end{array}$ & $\begin{array}{l}\text {-Las madres de familias } \\
\text {-Los padres de familias } \\
\text {-Agentes particulares } \\
\text {-Hermanos o tíos } \\
\text {-Ninguna persona les colabora, ellos tra- } \\
\text { bajan solos }\end{array}$ \\
\hline $\begin{array}{l}\text { CATEGORIA:COMPROMISOS } \\
\text { ESCOLARES } \\
\text { 7. ¿Cómo atienden a los padres } \\
\text { de familia, cuando por cualquier } \\
\text { motivo, acuden a la institución } \\
\text { educativa? }\end{array}$ & $\begin{array}{l}\text {-Con educación y agrado } \\
\text {-En forma puntual } \\
\text {-Con mucho respeto y cordia- } \\
\text { lidad }\end{array}$ & $\begin{array}{l}\text { Excelentemente atendido } \\
\text {-Son muy bien atendidos } \\
\text {-Con amabilidad responsabilidad } \\
\text {-Muy amablemente } \\
\text { Se explican inquietudes }\end{array}$ & $\begin{array}{l}\text {-La atención es muy buena, no hay quejas } \\
\text {-Siempre han atendido perfectamente } \\
\text {-Todos los docentes atienden bien } \\
\text {-Atención cordial con los padres de familia } \\
\text {-Muy respetuosamente. }\end{array}$ \\
\hline $\begin{array}{l}\text { 8. ¿De qué forma controlan la } \\
\text { asistencia de los estudiantes a la } \\
\text { institución y comoles colaboran } \\
\text { con los compromisos escolares } \\
\text { asignados? }\end{array}$ & $\begin{array}{l}\text { A través de llamada a lista. } \\
\text {-Registro de asistencia diario } \\
\text {-Llamando al acudiente para } \\
\text { justificar su ausencia. } \\
\text {-Revisando las tareas dejadas } \\
\text { por el docente }\end{array}$ & $\begin{array}{l}\text {-Me regañan sino asisto a clase } \\
\text {-Me aconsejan que el colegio es } \\
\text { lo mejor } \\
\text {-Siempre me llevan al colegio -A } \\
\text { través del registro de asistencia } \\
\text {-Siempre asisto a clase-Me obligan } \\
\text { a hacerlo }\end{array}$ & $\begin{array}{l}\text { Obligándolos a asistir al colegio } \\
\text {-Ellos van solo sin presión alguna } \\
\text {-Están pendiente que lleguen al colegio } \\
\text {-Los despiertan tempranoy les acompañan } \\
\text { a la institución } \\
\text {-El trabajo impide ejercer control }\end{array}$ \\
\hline
\end{tabular}

Fuente: Los autores 
PROPUESTA: El diseño de la misma, se constituyó en una matriz, qiue sería un guía, para los padres de fam,ilia, enfocada en la responsabilidad que los mismos tienen en la formación de sus hijos, y una herramienta para los educadores, enfocados en su compromiso con la formación de la niñez y la juventud en la institucion educativa Rural de Palmira, Pueblo-Viejo- Magdalena. Se espera que con ella, los padres cuenten con elementos para acompañar el proceso de formación de sus hijos. A través de esta propuesta de cambio, se permite a los padres de familias o responsables de los niños reflexionar colectivamente sobre la forma como pueden participar en el proceso educativo de sus hijos.

Para lo anterior se presentan los siguientes ejes temáticos:

- Cualificación a padres de familia

- Fortalecimiento de niveles de participación

- Fortalecimiento de canales de comunicación

- Normatividad sobre participación

Los mencionados ejes temáticos se organizaron mediante un plan de acción con sus etapas o fases, objetivos, actividades, responsables, recursos y resultados esperados que facilitan llegar a acuerdos de participación en la institución educativa. Dichas temáticas de interés fueron estructuradas teniendo en cuenta los resultados arrojados a partir del análisis de los instrumentos aplicados a los miembros de la comunidad educativa. (ver. Fig. 3)

\section{Conclusiones}

Al culminar todo el proceso investigativo el cual buscó "diseñar una estrategias educativa para la participación de padres de familia en los compromisos escolares de los estudiantes de grado quinto de la I.E.D. Rural de Palmira, Pueblo-Viejo, Magdalena", se pudo concluir que partir de la promulgación de la Constitución Nacional de(1991), la educación en nuestro país y región, ha generado una transformación en los diferentes estamentos educativos y muy especialmente en lo relacionado con la familia.

A pesar de lo manifestado en la carta magna, el grado de participación de los padres en el proceso educativo, no ha estado a la altura de lo contemplado en la norma, bien porque el sistema mismo no lo haya permitido o por un desconocimiento de sus deberes y derechos en este campo.

Como resultado de la aplicación de los instrumentos destacamos que generalmente el padre de familia asiste a la Institución a consultar sobre la situación académica de sus hijos, cuando son llamados por el docente o convocados por la institución. Hay

Fig. 3 Fortalecimiento del papel de la familia en la educación del niño

PROPUESTA EDUCATIVA

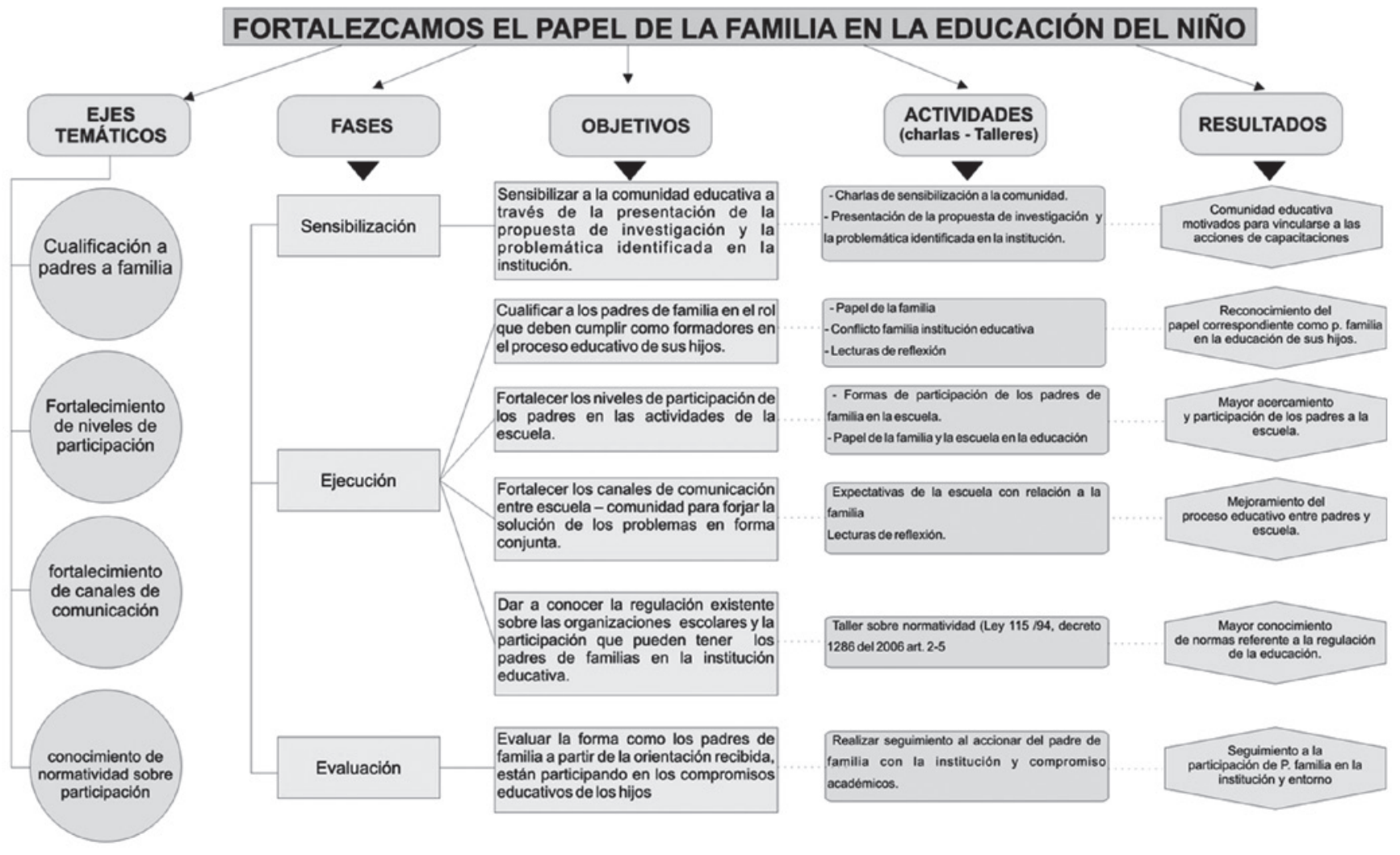

Fuente: Los autores 
padres de familia que en forma frecuente se acercan a pedir informes sobre el comportamiento de sus hijos o sobre su desempeño académico; lo cierto es que otros solo aparecen si alguien peleó o maltrató a su hijo, pero con la intención de defenderlo.

Las estrategias más utilizadas en busca de la participación de los padres de familias, es la información escrita enviadas con los estudiantes, pero estas solo se hacen cuando hay quejas de carácter académico o comporta mental del estudiante y cuando existen compromisos académicos que requieren la ayuda de los padres.

El nivel de responsabilidad que tienen los padres de familia con la educación de sus hijos es mínimo, y hay un desconocimiento por parte de ellos sobre su papel como formadores, lo que genera situaciones desfavorable en el contexto familiar para el desarrollo de los educandos. Así mismo es evidente la gran debilidad existente en los canales de comunicación entre los actores del proceso educativo, sumado esto a las falencias por parte de la institución en la creación de organismos integradores.

Dando respuesta a la formulación del problema, se concluyó que las estrategias educativas institucionales, para que los padres se vinculen a ella y colaboren en los compromisos escolares de sus hijos, en orden de importancia son: Mantener una relación constante con los padres de familias. Invitando al padre de familia a participar en los proyectos que se desarrollan, convocándolos a las reuniones, a través de invitaciones personalizada, e invitarlos a participar en las actividades realizadas en forma planificadas y no fortuita.

La propuesta da espacio para las siguientes recomendaciones: Que juntos, maestros y padres de familia encuentren mejores formas de participar en las actividades académicas de los estudiantes; para lo cual deben formular estrategias y condiciones para lograrlo.

Es necesario llevar a cabo unas acciones que permitan continuar con el acompañamiento permanente de los padres de familia en los propósitos y acciones de la escuela, además de seguir impulsando la conducta positiva de los padres de familia.

Abrir canales de comunicación e integración entre la familia y escuela para lograr beneficios en el acompañamiento de los compromisos escolares conjuntamente entre docentes y padres de familias.

Finalmente nombrar un personal que coordine y ponga en práctica la "Escuela de Padres" para involucrar los padres de familia y facilitar la participación activa y responsable de estos en los procesos educativos del estudiante.

\section{Referencias}

Alarcón, R. (2002). Creencias: Influencias en la Relación FamiliaEscuela. Seminario para optar al grado de Licenciado en Psicología. Universidad de Concepción
Arancibia, V., Herrera, P\& Strasser, K. (1999). Manual de Psicología Educacional Santiago: Pontificia Universidad Católica de Chile.

Buendía Eximan, Leonor, Pilar Colás Bravo y Fuensanta Hernández Pina (1999): Métodos de investigación en psicopedagogía. España. McGraw-Hill

Cide/Unicef. Centro de Padres [CEPA] E.B N 3 Domingo Matte. (1994). La Educación es tarea de todos. En Revista de Educación MINEDUC No 220, Septiembre, 2.

Cide - Unicef. (2000). Diálogo Social: Participación de los Centros de Padres en la Educación. Santiago de Chile:

Gallardo, L. y Calisto, R. (2004). Análisis comparativo del funcionamiento y el aporte de los equipos de gestión en liceos humanístico científico y técnico profesional de la comuna de Temuco. Visiones de la Educación, 5, 11 - 19.

González Bermúdez, J. (2012). La globalización en el siglo XXI y su impacto en la educación, la cultura y las habilidades. En: Contribuciones a la Economía.

Gubbins, V. \& Berger, C. (2001).Hacia una alianza efectiva entre familias y escuelas. Revista Persona y Sociedad, Instituto Latinoamericano de Doctrina y Estudios Sociales ILADES, Santiago.

Ley General de Educación (Ley 115 de 1994) recuperado de: www.mineducacion.gov.co

Martínez Torres, A. (2011), El papel de la familia y la escuela en la educación actual. Artículo enviado para su publicación en Profes.net, de la revista Actualidad Educativa,

Morales, F. (1998). Participación de Padres en la Escuela: Componente para la formación de profesores. Santiago de Chile: CIDE.

Morín, E. (2000). Los siete saberes necesarios a la educación del futuro. IESALC/UNESCO. Caracas, 2000.

Morín, Edgar. Introducción al Pensamiento Complejo. Barcelona, España, 2001a. (Trabajo original publicado en 1990).

Navarro, G. (2002). La participación de los padres en el proceso de enseñanza-aprendizaje de los hijos. Tesis Doctoral. Universidad de Concepción en cooperación con Universidad de Estocolmo.

Palacios, J. y Oliva, A. (1991): Ideas de Madres y Educadores sobre la Educación Infantil. MEC. Madrid.

Pineault, C. (2001). El desarrollo de la competencia de los padres. En Gervilla Castillo y otros. Familia y Educación. Grupo de investigación "Educación infantily formación de educadores". Universidad de Andalucía España. 